\title{
Erratum
}

\section{Organocatalytic Asymmetric Triple Domino Reactions of Nitromethane with $\alpha, \beta$-Unsaturated Aldehydes}

Dieter Enders,* Matthieu Jeanty, Jan W. Bats Synlett 2009, 3175.

The advance online publication (e-First) version of this article contained an error in Scheme 3.

This mistake has been corrected for both the current online version and the print publication. 